\title{
Following microscopic motion in a two dimensional glass-forming binary fluid
}

\author{
Matthew T. Downton ${ }^{1,2}$ and Malcolm P. Kennett ${ }^{1}$ \\ 1 Physics Department, Simon Fraser University, 8888 University Drive, Burnaby, \\ British Columbia, V5A 1S6, Canada \\ 2 Institut für Theoretische Physik, Technicshe Universität Berlin, 10623 Berlin, \\ Germany
}

\begin{abstract}
The dynamics of a binary mixture of large and small discs are studied at temperatures approaching the glass transition using an analysis based on the topology of the Voronoi polygon surrounding each atom. At higher temperatures we find that dynamics is dominated by fluid-like motion that involves particles entering and exiting the nearest-neighbour shells of nearby particles. As the temperature is lowered, the rate of topological moves decreases and motion becomes localised to regions of mixed pentagons and heptagons. In addition we find that in the low temperature state particles may translate significant distances without undergoing changes in their nearest neighbour shell. These results have implications for dynamical heterogeneities in glass forming liquids.
\end{abstract}




\section{Introduction}

The relationship between the dynamics and structure of a glass-forming liquid is a non-trivial problem. Structurally, a simple liquid is isotropic and has smooth pair distribution functions that decay to the background value within a few nearest neighbour spacings. The positions of the maxima and minima of these functions can be explained in terms of coordination shells of particles packed around a central reference particle. This gives an intuitive understanding of the microscopic structure of a liquid. The corresponding picture of dynamics is based around atoms that are trapped by their surrounding neighbours, and make rare "cage-breaking" jumps to nearby positions. At high temperatures, the separation in time between collisions with neighbours and jumps is small, but at lower temperatures this time starts to diverge. How different arrangements of atoms promote or inhibit this motion is an open question.

Molecular dynamics simulations provide a powerful tool to address this problem by allowing one to follow structure and dynamics simultaneously. It has been observed in simulations that systems that exhibit glass-like behavior at lower temperatures can have characteristics of an interplay between crystal and liquid structure [1. Structural changes have also been observed in fluids approaching the fluid-solid transition [2]. Additionally, it has been observed that as the glass transition is approached, dynamics become increasingly spatially heterogeneous [3, 4, 5, 6, 17, 8, 19, 10, 11, 12, 13, 14]. The character of these dynamical heterogeneities has been well studied, both computationally [13, 15, 16, 17, 18, 19, 20] and in experiments on colloidal glasses [21, 22], in which "caging" of particles, and the escape from cages via string-like motion has been seen at temperatures slightly above the glass transition temperature. Despite all of this effort, the origin of dynamical heterogeneities is unclear, as is their connection to the dramatic slowing down of dynamics seen as a liquid becomes glassy.

A simulation approach that has been recently introduced, the iso-configurational

ensemble, sheds light on the relationship between structure and dynamics [23]. In this technique, repeated short simulations are performed using the same starting structure, but randomized velocities. Averages of single particle displacements over the ensemble of simulations give a map of the propensity to move. It has been observed that the local Debye-Waller factor appears to correlate very closely with the dynamic propensity at long times [24], however it is harder to answer the more subtle question of how the propensity correlates with the dynamics of a single trajectory. It appears that on the single-particle level, such a connection cannot be made [25], but that the connection between structure and dynamics will exist at longer lengthscales.

An alternative theoretical approach to understanding the behaviour of glass-forming liquids, and in particular their kinetic arrest in the vicinity of the glass transition temperature, has been through studying phenomenological models. Such models, in particular, kinetically constrained models (KCMs) have received considerable attention in recent years [26, 27, 28, 29, 30, 31, 32, 33, as they have had success in reproducing diverging timescales observed in glass-formers. These models are generally understood 
as an effective model for some coarse-grained degree of freedom (often referred to as spins), and at low temperatures, the dynamics can be understood as a few up-spins or "defects" in a background sea of down spins [34]. We have recently attempted to provide a coarse-graining procedure to map the dynamics of a glass-former onto a KCM, using local mean squared displacement as a means to define spins [35. This approach found kinetically constrained behaviour became increasingly important at low temperatures, but only on timescales longer than the alpha relaxation time, whereas one would hope that the alpha relaxation time was in fact a consequence of the KCM, rather than an input. For shorter coarse-graining times, there was no evidence of kinetically constrained dynamics. This suggests that alternative defect variables should be identified if such a coarse-graining procedure is to be successful.

In this paper we examine the interplay between structure and dynamics by investigating the dynamics of a two-dimensional liquid in terms of the coordination number given by a Voronoi polygonisation. Previously, the population densities of different coordinations have been used to make predictions regarding the glass transition temperature 36. Here we use this choice to give an unambiguous definition of 'fluidlike' motion within a dense particle system in terms of a simple topological process. The approach we take can also be related to previous work on lattice based models that obey a similar dynamical rule [37, 38, 39]. We find that there are a few dominant particle moves, of which, only one has significant temperature dependence, becoming relatively more important with decreasing temperature.

The structure of the paper is as follows: in Sec. 2 we give details of the model and simulation procedures, along with some results that show the development of glassy phenomenology at lower temperatures. In Sec. 3 we define the "defects" we consider and discuss their spatial distribution, and in Sec. 4 we present the microscopic dynamics of the fluid in terms of local topology changing transitions. These results are followed by concluding observations and discussion in Sec. 5 .

\section{Model and simulation details}

We used a two-dimensional model that exhibits all of the key ingredients of a true glassformer. The model consists of a binary mixture of large and small soft discs with size ratio 1:1.4 interacting with a potential of the form $V_{\alpha \beta}=\epsilon\left(\frac{\sigma_{\alpha \beta}}{r}\right)^{12}$. The mass, $m$, of all particles was set to unity; time was measured in units $\tau=\sqrt{m \sigma_{11}^{2} / \epsilon}$; distance was measured in terms of the small particle diameter, $\sigma_{11}$; temperature was measured in units of $\epsilon$.

Molecular dynamics simulations of 1600 particles in the ratio $75: 25$ small to large were performed at constant volume and temperature with the number density fixed to $0.85 \sigma_{11}^{-2}$. Configurations were equilibrated at high temperatures then slowly cooled to create a series of configurations at different temperatures. These were then extensively equilibrated at fixed temperature using a Gaussian isokinetic thermostat. At temperatures below $T=0.330$ the system was found to age as indicated by a continuous 

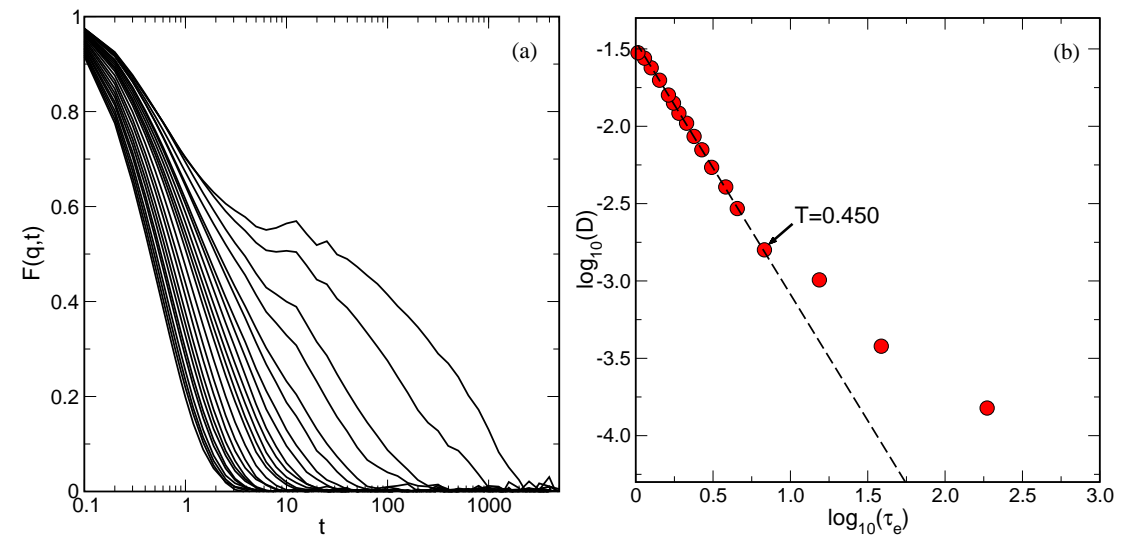

Figure 1. Development of glassy behavior at lower temperatures. (a) The dynamic structure factor, $F(q, t)$, calculated at $q=6.23 \sigma^{-1}(T=0.360-1.440)$ (b) Change in scaling of the structural relaxation time, $\tau_{\alpha}$, and the diffusion coefficient for small particles.

decrease in the pressure and total potential energy. In a separate series of calculations it was found that when the initial configuration consisted of segregated small and large particles, there was complete mixing for temperatures $T \geq 0.450$; below this temperature there was no mixing for initially segregated configurations other than relaxation at the phase boundary.

The phenomenology of this mixture is almost identical to the mixture studied by Perera and Harrowell: multiple relaxational timescales develop at lower temperatures; a change in the scaling between structural relaxation and diffusion occurs at a temperature $T^{*}$ [40, 41, 42]. These results are summarised in figure 1 where we take the structural relaxation time, $\tau_{\alpha}$, to be the time it takes the function

$$
F(q, t)=\frac{\langle\rho(\mathbf{q}, t) \rho(-\mathbf{q}, 0)\rangle}{\langle\rho(\mathbf{q}, 0) \rho(-\mathbf{q}, 0)\rangle},
$$

to decay to the value $1 / e$, where $\rho(\mathbf{q}, t)=\sum_{j} \exp \left(i \mathbf{q} \cdot \mathbf{r}_{j}\right)$ and $q=|\mathbf{q}|$ is the position of the first peak in the static structure factor. Similar results were found if the self-intermediate scattering function for a single particle species was used to measure structural relaxation. It can be seen that $T^{*} \approx 0.450$ for this particular mixture: at temperatures below $T^{*}, F(q, t)$ begins to exhibit two stage decay.

\section{Static results}

\subsection{Distribution and concentration of defects}

The Voronoi procedure partitions the system into polygonal cells surrounding each atom. Nearest neighbours are identified as atoms which share an edge, whereas next-nearest neighbours have common nearest neighbours but are not themselves neighbours. For convenience we refer to atoms with coordination number, $c$, differing from six as defects and use the "topological charge", $q=c-6$, to characterise the coordination of individual 


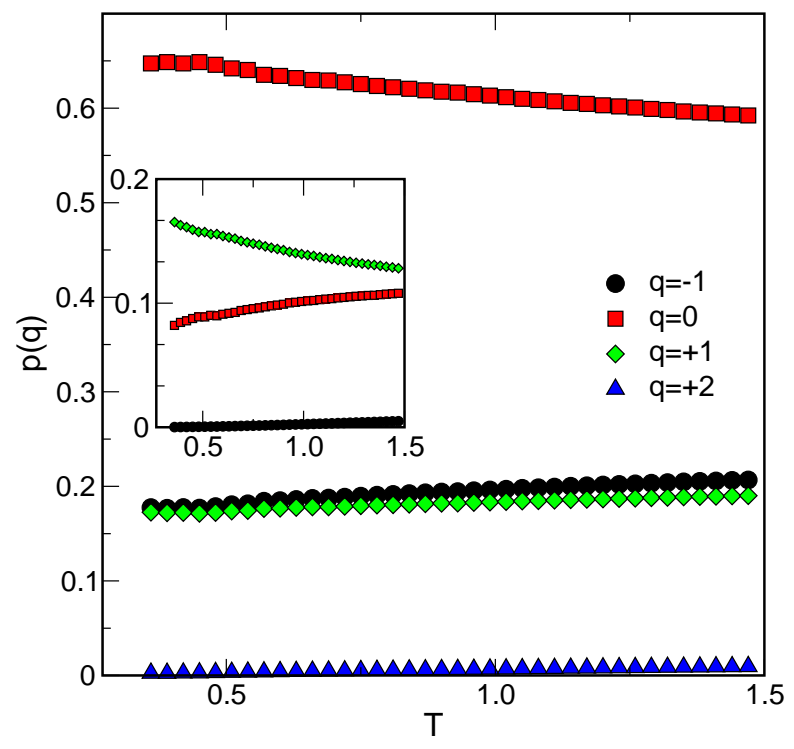

Figure 2. Population of defect species as a function of temperature in the non-aging temperature range. Inset: Topological charge of large atoms as a proportion of the total number of atoms.

atoms. Within simulations such as this where periodic boundary conditions are used, the total coordination is conserved with $\sum_{i} q_{i}=0$.

The variation with temperature of the concentration of atoms with different $q$ is shown in figure 2, Although occasional configurations contained four-fold or nine-fold coordinated atoms these were found to be relatively rare and the majority of atoms have $q$ in the range -1 to +2 . At higher temperatures there is an excess of -1 atoms to +1 atoms that is compensated for by a small number of +2 atoms. At all temperatures within the range of study, the majority of large atoms have charge +1 and these constitute almost all of the +1 atoms at the lower end of this range (inset to figure 2). The number of $q=0$ atoms also increases as a function of $T$, before levelling out at $T^{*}$.

Two configurations representative of high and low temperatures are shown in figure 3. In both configurations, the distribution of large and small particles is similar and there is no apparent demixing of the system at lower temperatures. However, a greater degree of hexagonal coordination is seen within the lower temperature configuration; this is especially noticeable in larger domains of small particles. Accompanying these snapshots are pictures of the system showing the positions of atoms with non-zero $q$. For $T=0.870$ there is a slightly higher concentration of lone defects. In both systems there is a network of defects which almost spans the system. Inspection of figure 2 shows that in fact at the temperature at which the Stokes-Einstein relation breaks down, $T^{*}$, the population of $q=0$ sites reaches $\sim 0.65$, and the threshold for bond percolation on a triangular lattice is 0.347 [43]. Relationships between percolation and breakdown of the Stokes-Einstein relation have been noticed by earlier authors in the context of a lattice gas [44] and a three dimensional glass former [45]. The defect network is more tightly connected at lower temperatures, and leaves larger regions that 


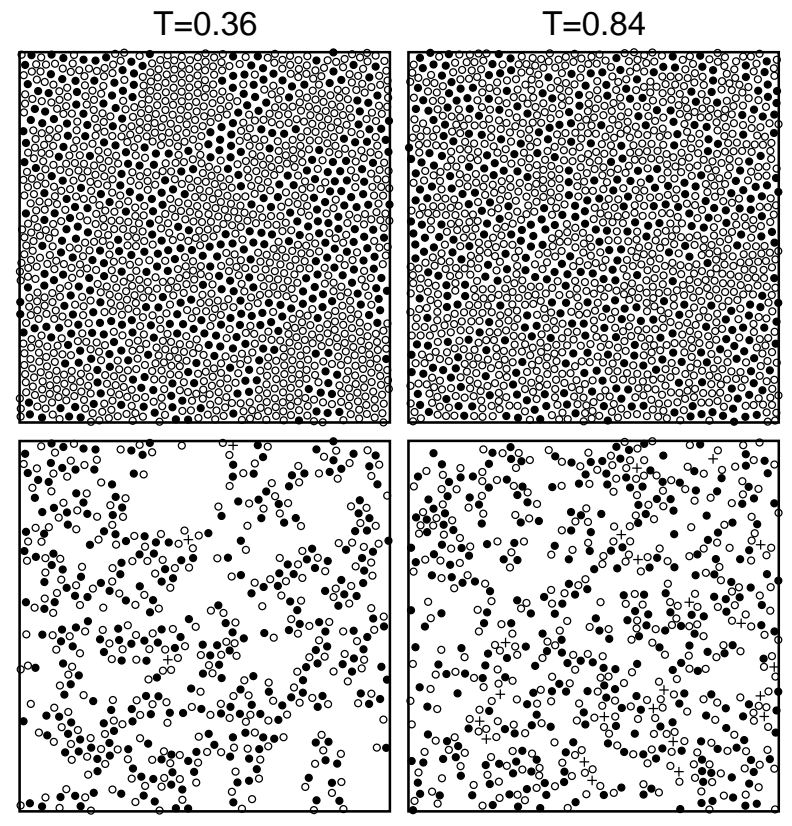

Figure 3. Particle positions and coordination at high and low temperatures. From left to right: $T=0.36$ and $T=0.84$. The top configuration shows the spatial distribution of large and small particles as filled and open circles respectively. In the lower configuration, atoms with $q=+1$ are filled circles, atoms with $q=-1$ are open circles, atoms with $q=+2$ are marked with a $(+)$.

are defect free and consist of small particles. Defects are generally arranged in pairs, so that $\mathrm{a}+1$ defect is the neighbor to a -1 defect; no net separation of charge can be observed at either high or low temperatures.

In figure 4 we plot the temperature dependence of the autocorrelation function of $q$. This is defined as

$$
C_{i}(t)=\frac{\left\langle q_{i}(t) q_{i}(0)\right\rangle-\left\langle q_{i}\right\rangle^{2}}{\left\langle q_{i}^{2}\right\rangle-\left\langle q_{i}\right\rangle^{2}} .
$$

At high temperatures $C(t)$ decays rapidly before plateauing and then decaying more slowly. Reducing the temperature gives rise to a more complicated decay pattern and a second long-time decay rate appears that grows at a similar rate as the dynamic structure factor, $F(q, t)$. Hence the dynamics of structural "defects" clearly mirrors that of density correlations, suggesting that there may be some connection between the two.

\section{Topology based dynamics}

One output of a Voronoi tesselation is a graph of nearest neighbours within the system. If fluid-like motion in real space can be thought of as the random motion of particles between the nearest and next-nearest neighbour shells then this motion has a simple topological rule that governs the creation and destruction of edges on the graph or equivalently the changes in the number of edges on each Voronoi cell. These dual views 


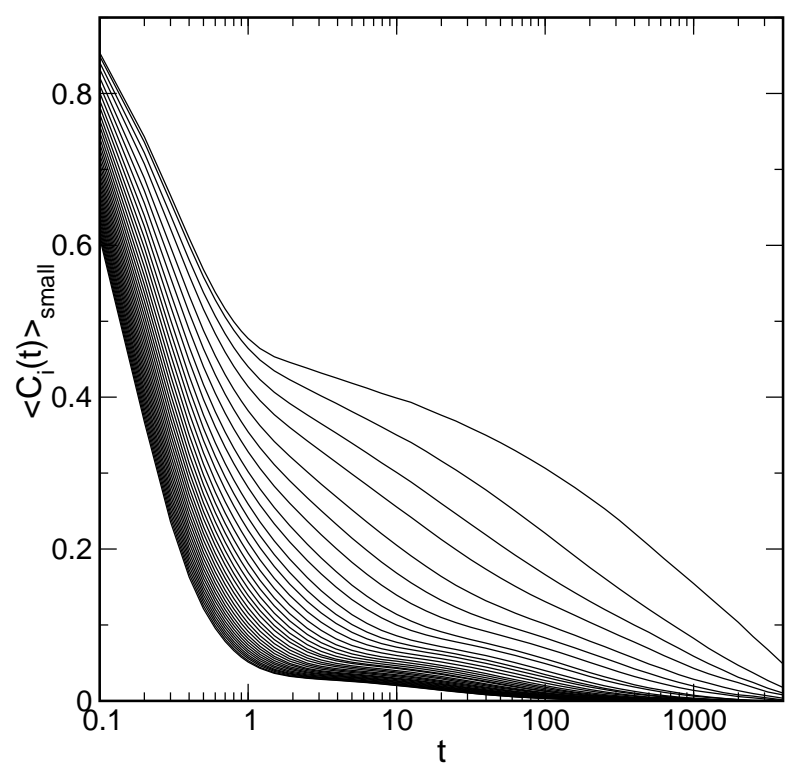

Figure 4. Autocorrelation function of topological charge averaged over small atoms. From top: temperatures in the range $T=0.360-1.470$ in steps of 0.03 .

of the dynamics are shown in figure 5. It is clear from the figure that pairs of particles that become nearest neighbours each gain an edge from the displaced particles. Provided that the number of particles is conserved, this single rule can be used to describe all of the changes in the coordination that occur during a simulation or experiment [46. This gives a suitable coarse-graining of configurational changes and allows us to examine the microscopic origin of the decay of the topological charge found in the previous section.

In the following section we proceed as follows: A standard molecular dynamics simulation is performed and the Voronoi procedure is performed frequently enough that the majority of nearest neighbour changes can be directly identified $t$. We then examine the frequency with which moves occur, the type and spatial distribution of nearest neighbour changes and finally examine the relationship with particle displacements at low temperatures. In the present study we ignore the identity of the atoms.

\subsection{Identity and spatial distribution of nearest neighbour exchanges}

The majority of nearest neighbour exchanges consist of the six basic moves listed in table 1. In this table, we have listed the topological charge of each atom before the nearest neighbour exchange occurs. The atoms in each case are labelled according to figure 5: $i$ and $l$ are acceptor atoms that each gain an extra nearest neighbour after the T1 move; $j$ and $k$ both lose nearest neighbours after the exchange. Many other exchange types are observed in the simulations, but are relatively infrequent in comparison to those listed.

The temperature dependence of the relative frequency of these move types is plotted

$\ddagger$ In practice, with a timestep of $\Delta t=0.0005 \tau, 94 \%$ of $\mathrm{T} 1$ moves can be identified at $T=0.840$ and $98 \%$ at $T=0.360$. The remaining moves are groups of $5-8$ atoms that change coordination simultaneously. 

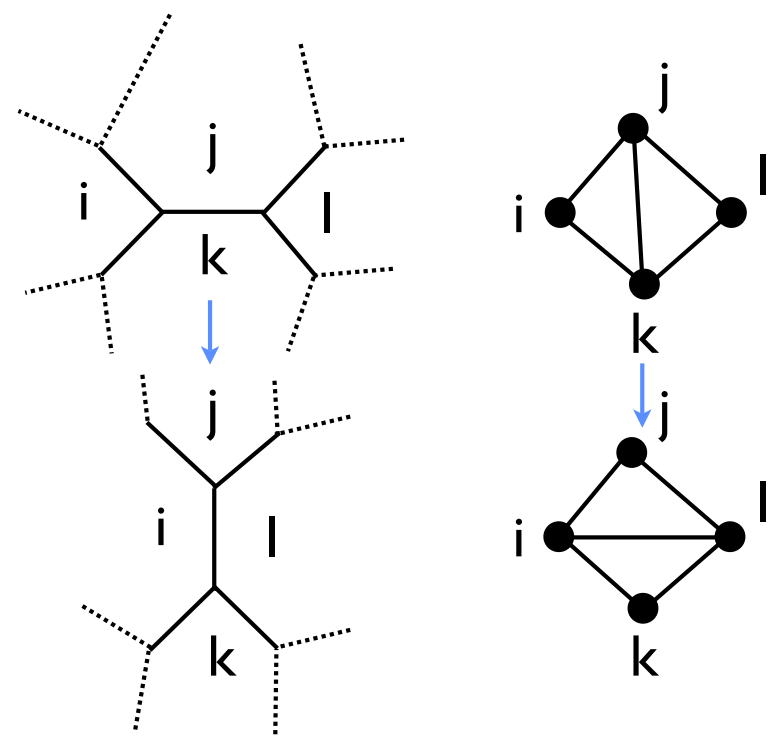

Figure 5. The T1 move in both representations of the particle positions (a) from the Voronoi cells (b) from the Delaunay triangulation of nearest neighbours. In these dual views, $i$ and $l$ move together to become nearest neighbours, $j$ and $k$ are pushed apart, becoming next-nearest neighbours. All other neighbours of the four atoms remain the same.

in figure 6(a). Two aspects of the dynamics are immediately apparent. First, there is no sudden change in the relative frequency of any particular T1 move at the temperature where the change in scaling between diffusion and structural relaxation occurs. Second, moves which are the inverse of one another are found to occur with similar frequency. This can be seen as an indication that detailed balance holds for the liquid and that the simulations are in equilibrium on the time scales studied. In figure 6(b), the rate at which T1 moves are observed is plotted as a function of temperature and can be seen to be surprisingly linear.

At all temperatures, the most significant move is the dislocation glide (move $\mathbf{1}$ ) where a pair of +1 and -1 defects translate by a single particle spacing in a direction that is roughly perpendicular to the line segment that connects the two atoms. The frequency of this move has been noted in previous simulation studies of liquid dynamics [47. The move $\boldsymbol{2}_{\text {a }}$ involves the combination of a -1 defect with three atoms that have coordination of six to produce two -1 defects, a single +1 defect and a six-fold coordinated atom. $\mathbf{2}_{\mathrm{b}}$ is the reverse of this move. This pair of moves is therefore one route by which a lone \pm 1 defect pair may be created or annihilated and is the only move of the most frequent that shows any strong temperature dependence. The final move that we consider in detail is the pair $\mathbf{3}_{\mathrm{a}}$ and $\mathbf{3}_{\mathrm{b}}$. This involves the destruction of two +1 and two -1 defects in one direction or the creation of these defects in the other direction and will play an important role in the melting and annealing of crystal lattices.

Having identified moves as they occur, the next stage in understanding the dynamics of the liquid is to examine the spatial distribution of nearest neighbour exchanges. In figure 7(a), we show the distribution of the first $1000 \mathrm{~T} 1$ moves observed after the 


\begin{tabular}{rrrrrrrrrrrr}
\hline & $i$ & $j$ & $k$ & $l$ & $c$ & & $i$ & $j$ & $k$ & $l$ & $c$ \\
\hline $\mathbf{1}$ & -1 & 1 & 0 & 0 & 0 & $\mathbf{4}_{\mathrm{a}}$ & -1 & 1 & 1 & 0 & 1 \\
$\mathbf{2}_{\mathrm{a}}$ & -1 & 0 & 0 & 0 & -1 & $\mathbf{4}_{\mathrm{b}}$ & 0 & 0 & 1 & 0 & 1 \\
$\mathbf{2}_{\mathrm{b}}$ & -1 & 0 & 1 & -1 & -1 & $\mathbf{5}$ & -1 & 0 & 0 & -1 & -2 \\
$\mathbf{3}_{\mathrm{a}}$ & -1 & 1 & 1 & -1 & 0 & $\boldsymbol{6}_{\mathrm{a}}$ & -1 & 0 & 0 & 1 & 0 \\
$\mathbf{3}_{\mathrm{b}}$ & 0 & 0 & 0 & 0 & 0 & $\boldsymbol{6}_{\mathrm{b}}$ & -1 & 0 & 2 & -1 & 0 \\
\hline
\end{tabular}

Table 1. Processes that comprise most of the nearest neighbour exchanges observed in the simulations. The columns $i, j, k$ and $l$ use the same atom labels as figure 5 and give the topological charge on each atom before the exchange occurs. The number in bold refers to the label used in figure 6. Exchanges which are the mutual reverse of one other are labelled with the subscripts $a$ and $b$.
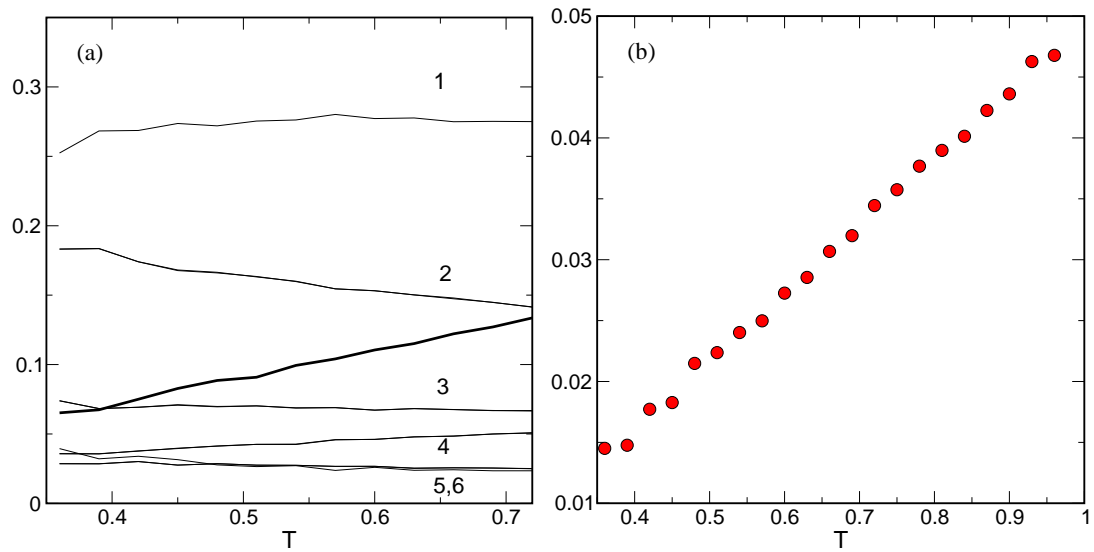

Figure 6. (a) Temperature dependence of the probability of the six most frequent T1 moves. The labelled curves refer to the moves in listed table 1 the thick solid line indicates the probability of all other moves. For moves 2, 3, 4 and $\mathbf{6}$ both sets of curves are plotted, but are almost indistinguishable from one another. (b) Number of T1 moves per particle per unit time.

$T=0.360$ snapshot shown in figure 3 was taken. These are strongly localised around the mixed regions of +1 and -1 defects that were previously observed in figure 3. Figure 7 (b) shows that there is not a strong correlation between the number of T1 moves observed for a given particle and the distance that it translates. In fact there seem to be a number of particles that displace relatively large distances during the short simulations without any T1 moves. However, the maximum displacement for particles that experience large numbers of T1 moves does tend to be smaller than those with fewer T1 moves.

One problem with the method that we present here is that we have no simple way of accounting for quartets of atoms are in almost square conformations. Small fluctuations around particle positions could then lead to the mis-counting of a large number of T1 moves. To address this we have repeated the simulations and changed the frequency with which the Voronoi procedure is performed and do not find any changes in the overall rate of T1 moves. Such counting problems are therefore unlikely to be significant. 

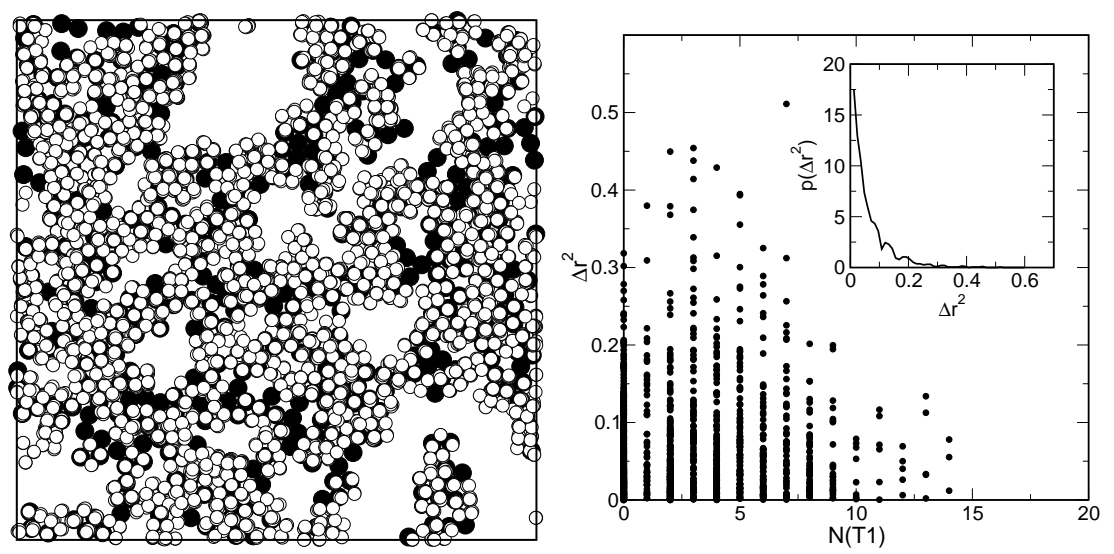

Figure 7. (a) Distribution of $1000 \mathrm{~T} 1$ moves (white circles) at $T=0.360$ superimposed on the positions of the large atoms in the starting configuration (black circles) (b) Correlation between the number of $\mathrm{T} 1$ moves that an atom undertakes and the mean squared displacement, $\Delta r^{2}$ (inset) Probability distribution function of $\Delta r^{2}$.

\section{Conclusions}

We have presented results of simulations of a two dimensional glass-forming binary fluid that shows identifiable changes in its structure when the control temperature is lowered. These changes are related to the spatial distribution of atoms with coordination that differs from the mean value. Using the topological properties of the two-dimensional nearest neighbour network we have shown that it is possible to identify a strong relationship between one aspect of the spatial distribution of dynamics and the local level of disorder in structure. There is apparently still motion within the simulation cell that is not accounted for by the topological process that we study, but our work may contribute to understanding of the puzzle presented by heterogeneous dynamics in slow and glassy systems. The observation that the rate of $\mathrm{T} 1$ moves decreases linearly with temperature, with the relative proportions of different types of T1 moves fairly insensitive to temperature, suggests that the slowing down associated with the glass transition is likely not to be able to be ascribed to any one set of $\mathrm{T} 1$ processes. Recent work has suggested that a particular class of "liquid-like" defects (which presumably have some set of associated topological moves) may play a role in the glass transition [36], but we have not explored this possibility here. However, the temperature dependence of the time decay of the correlations in topological charge, and its similarity to the decay of the dynamic structure factor suggest that the dynamics of T1 moves may be relevant to dynamic facilitation as is required for kinetically constrained models of glasses.

\section{Acknowledgments}

Michael Plischke is thanked for encouragement and helpful discussions. NSERC and Westgrid are thanked for research funding and computing facilities. 


\section{References}

[1] Shintani H and Tanaka H 2006 Nature Phys. 2200

[2] Truskett TM, Torquato S, Sastry S, Debenedetti PG and Stillinger FH 1998 Phys. Rev. E 583083

[3] Sillescu H 1999 J. Non-Cryst. Solids 24381

[4] Ediger MD 2000 Annu. Rev. Phys. Chem. 5199

[5] Glotzer SC 2000 J. Non-Cryst. Solids 274342

[6] Richert R 2002 J. Phys.: Condens. Matter 14 R703

[7] Castillo HE, Chamon C, Cugliandolo LF and Kennett MP 2002 Phys. Rev. Lett. 88237201

[8] Vidal-Russell E and Israeloff NE 2000 Nature 408695

[9] Deschenes LA and Vanden Bout DA 2001 Science 292255

[10] Reinsberg SA, Qiu XH, Wilhelm M, Spiess HW and Ediger MD 2001 J. Chem. Phys. 1147299

[11] Weeks ER and Weitz DA 2002 Phys. Rev. Lett. 89095704

[12] Kob W, Donati C, Plimpton SJ, Poole PH and Glotzer SC 1997 Phys. Rev. Lett. 792827

[13] Donati C, Douglas JF, Kob W, Plimpton SJ, Poole PH and Glotzer SC 1998 Phys. Rev. Lett. 80 2338

[14] Donati C, Glotzer SC, Poole PH, Kob W and Plimpton SJ 1999 Phys. Rev. E 603107

[15] Perera DN and Harrowell P 1996 Phys. Rev. E 541652

[16] Yamamoto R and Onuki A 1998 Phys. Rev. Lett. 814915

[17] Andersen HC 2005 Proc. Nat. Acad. Sci. 1026686

[18] Perera DN and Harrowell P 1998 J. Non-Cryst. Solids 235314

[19] Gebremichael Y, and Vogel M and Glotzer SC 2004 J. Chem. Phys. 1204415

[20] Stevenson JD, Schmailian J and Wolynes PG 2006 Nature Phys. 2268

[21] Kegel WK and van Blaarderen A 2000 Science 287290

[22] Weeks ER, Crocker JC, Levitt AC, Schofield A and Weitz DA 2000 Science 287627

[23] Widmer-Cooper A, Harrowell P and Fynewever H 2004 Phys. Rev. Lett. 93135701

[24] Widmer-Cooper A and Harrowell P 2006 Phys. Rev. Lett. 96185701

[25] Berthier L and Jack RL 2007 Phys. Rev. E 76041509

[26] Ritort F and Sollich P 2003 Adv. Phys. 52219

[27] Fredrickson GH and Andersen HC 1984 Phys. Rev. Lett. 531244

[28] Fredrickson GH and Andersen HC 1985 J. Chem. Phys. 835822

[29] Graham IS, Piché L and Grant M 1997 Phys. Rev. E 552132

[30] Garrahan JP and Chandler D 2002 Phys. Rev. Lett. 89035704

[31] Berthier L and Garrahan JP 2003 J. Chem. Phys. 1194367

[32] Garrahan JP and Chandler D 2003 Proc. Nat. Acad. Sci. 1009710

[33] Léonard S, Mayer P, Sollich P, Berthier L and Garrahan JP 2007 J. Stat. Mech.: Theory Exp. 07017

[34] Berthier L and Garrahan JP 2003 Phys. Rev. E 68041201

[35] Downton MT and Kennett MP 2007 Phys. Rev. E 76031502

[36] Aharonov E, Bouchbinder E, Hentschel HGE, Ilyin V, Makedonska N, Procaccia I and Schupper N 2007 Europhys. Lett. 7756002

[37] Davison L and Sherrington D 2000 J. Phys. A: Math. Gen. 338615

[38] Davison L, Sherrington D, Garrahan JP and Buhot A 2001 J. Phys. A: Math. Gen. 345147

[39] Sherrington D, Davison L, Buhot A and Garrahan JP 2002 J. Phys.: Condens. Matter 141673

[40] Perera DN and Harrowell P 1998 Phys. Rev. Lett. 98120

[41] Perera DN and Harrowell P 1999 Phys. Rev. E 595721

[42] Perera DN and Harrowell P 1999 J. Chem. Phys. 1115441

[43] Sykes MF and Essam JW 1964 J. Math. Phys. 51117

[44] Nicodemi N and Coniglio A 1998 Phys. Rev. E 57 R39

[45] Dzugutov M, Simdyankin SI and Zetterling FHM 2002 Phys. Rev. Lett. 89195701

[46] Larson RG 1999 The Structure and Rheology of Complex Fluids (Oxford: Oxford University Press) 
[47] Deng D, Argon AS and Yip S 1989 Phil. Trans. R. Soc. Lond. A 329575 